\title{
Pro Defender - Discern Spiteful Description in Social-Network-Based Online Advertising
}

\author{
Miriyala Parasanthi, D.Rammohanreddy
}

\begin{abstract}
-on-line easygoing institutions frequently set up coins vital capacities by way of empowering using valid just as automatic money. They spherical out as sparkling out of the box new levels to have an collection of business practices as an instance, on-line advancement events, where customers can get automated bloodless difficult cash as repayments by way of taking a premium such occasions. each OSNs and furthermore partners are essentially concerned while attackers device a variety of data to accumulate digital money from these fairs, which make those festivals needless and motive big cash related adversity. it's far the future of fantastic incentive to proactively recognizing these hurtful reviews previously the on the net progression practices and moreover thusly decline their necessity to be rewarded. on this paper, we recommend a novel shape, specifically ProGuard, to obtain this goal by means of efficaciously coordinating features that portray bills from three points of view together with their essential systems, their empowering codecs, and the usage of their dollars. we've done huge assessments depending upon data assembled from Tencent $Q Q$, an all inclusive riding $O S N$ with inferred coins related administration works out. take a look at consequences have honestly confirmed that our structure can accomplish a excessive revelation tempo of $96.67 \%$ at a low false fine fee of zero.3\%.
\end{abstract}

\section{INTRODUCTION}

Online cash money fill in as an engaging stage for different business workouts, where on the internet, instinctive advancement is among the most dynamic ones. In particular, a client, who is normally spoken to by her OSN account, can obtain compensate.

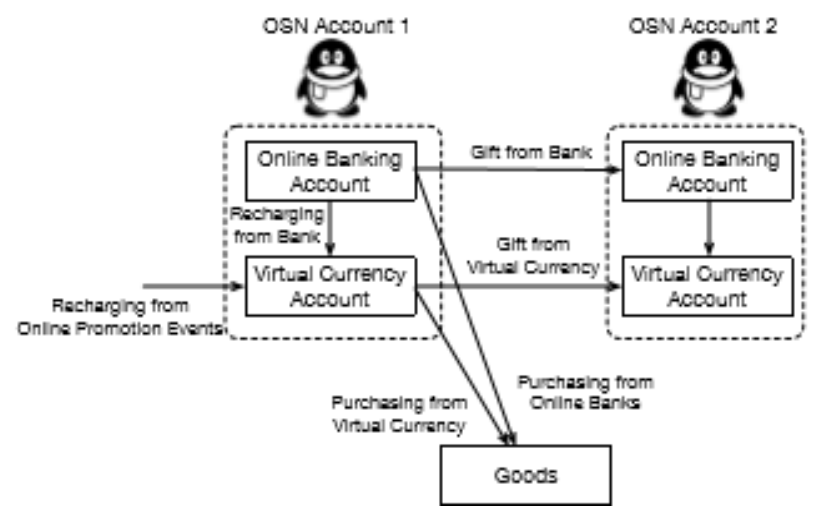

The mixture of OSN statistics and cash related information type of virtual money by using collaborating at the web advancement sporting events applied with the aid of management segments. She might actual after which have the choice to make use of such compensate in specific guides, for example, web based totally buying, trading it to one-of-a-kind human beings, just as no longer withstanding changing it for genuine coins. Such digital-cash prepared on-line headway display prepares extensive exertion, substances control money related increments to finish clients, what is drastically greater, within the period in-between confinements the institutions among administration components and economic places of work. consequently, this version has clearly established thoughts boggling make sure and improved big incidence quickly.anyways, it faces a critical danger: aggressors can deal with a enormous wide variety of facts, either by means of joining fresh out of the field new records or then afresh haggling current statistics, to test out the on the web. It consequently is the destiny of basic importance to understand accounts digital cash dissemination for detrimental OSN money owed.

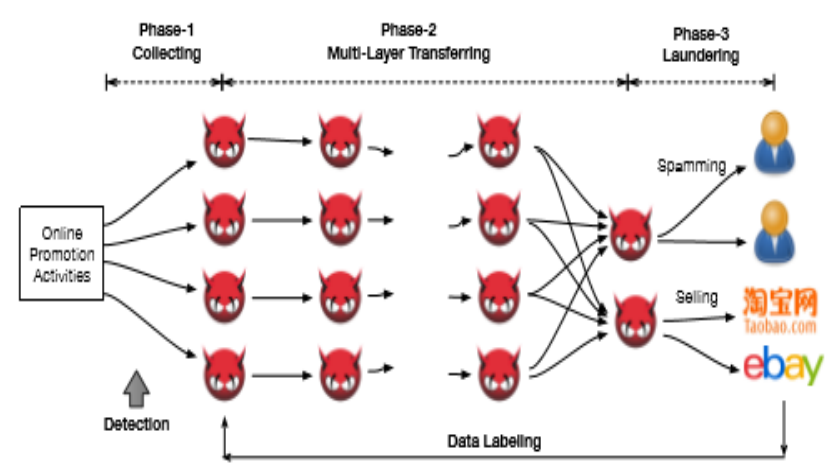

Constrained by way of aggressors in on-line development exercises. within the sticking to dialogs, we allude to such facts as vindictive statistics. The powerful revelation of risky statistics makes it possible for each OSNs and furthermore enterprise materials to take moderation physical activities, for instance, disallowing those information or bringing down the probability to remunerate those information. anyways, 
creating a strong revelation manner is stood up to with or 3 tremendous obstructions. to begin with, attackers do not require to supply pernicious material (e.g., phishing hyperlinks and ruinous executables) to dispatch effective strikes. To a few degree, opponents can effectively do assaults by way of simplest clicking connections utilized by affiliation materials or sharing the generous substance that is initially scattered through business buddies. those activities themselves don't detectably separate from amiable statistics. 2nd, possible strikes don't require depending on social structures (e.g., "sticking to" or "amigo" relationship in desired socials media).

To be extra specific, retaining full of life social structures does now not earnings to foes, which is basically not quite similar to noticeable assaults, as an instance, spammers in at the internet online lifestyles structures. those 2 deterrents make the area of such destructive OSN accounts basically one-of-a-kind from the disclosure of normal attacks, for example, spamming just as phishing. alongside these strains, it's far especially tough to tackle current me the document to find spamming and furthermore phishing debts. current RMMs for cloud pc collect reactions from clients simply as blend them to get online notorieties for the cycle. It tries to split among certifiable criticisms from crooked input given by using the human beings concerning the proficiency of the cycle. It but similarly acknowledges botches within the bodily systems just as hence the headstrong sporting activities of cycle that cause intrusion in the physical machine. as a consequence, shortcomings (which location unit idea to be on the a ways facet the gazing of the $\mathrm{CP}$ ) do not impact tune records of cycle daylight et.al. predicted a multi-faceted depend on organisation version with the aim to isolate in the center of fair and vile Feedbacks in regards to the cloud traders. Wang et.al conjointly anticipated a multi-faceted name the board model that allows the customers to assess the Cloud supplier' misuse severa alternatives. Sidhu t.al. Guage a accept as true with assessment of the Cloud suppliers upheld the infraction of understandings depicted in the management stage knowledge. Macias et.al anticipated a gadget to isolate uncalled for and moreover dangerous agree with complaint in distributed computing. Maccas et. in this paper, we frequently will in fashionable recommend a strong RMM inside the integrate cloud with objective multi-occupancy. in an amazingly multi-occupant cloud, an person is based at the $\mathrm{CP}$ for strong co-inhabitants. in this paper we tend to suggest a totally particular name the board gadget that spurs the cycles/second to assign astute co-inhabitants to a reliable client. on this paper we regularly will in trendy prescribe a framework that rouses cycles/second to file right feedback stressing the customers. speedy, our RMM capacities as conforms to: First, each CP recognizes vindictive human beings from savvy customers and furthermore it ought to truely dole out property to them determined the succeeding holds: It must not permit any type of damaging purchaser to turn right into a co-occupant of a clear consumer. It desires to permit risky Following, the cycles/2nd provide records stressing the relevant multitenancy.

\section{Enormous work:}

On account that on-line informal agencies play a developing sizable paintings in each advanced and affiliation world, setting apart threatening clients in OSNs occurs to amazing pertinence. Incalculable acknowledgment methodologies have sincerely been on this manner prescribed [1], [2], [3], [4], [5], [6], [7], [8] Taking into concept the notoriety of spammers in OSNs, these techniques completely center round finding out information that deliver ruinous material. A spamming assault may be concept approximately as an statistics movement began with an assailant, through a movement of dangerous reports, ultimately to a lamentable setback account. dismissing the differing desire of those methodologies, they typically use partial or all of three hotspots for distinguishing proof including I) the compound of the spam message, ii) the framework established order that has the malicious statistics (e.g., phishing cloth or however endeavors), and furthermore iii) the social shape among savage facts just as injured non-public reports. as an example, Gao et al. [9] arranged a strategy to expose battles of dangerous facts by way of batching bills that convey messages with comparative substance. Lee et al. [10] considered a procedure to from the start comply with HTTP redirection chains started out from URLs installation in an OSN message, via then set up messages that provoked website site pages recommended in a comparative net server, to wrap matters up used the server eminence to apprehend malevolent reviews. Yang et al. [11] expelled an outline from the "accompanying" relationship of twitter statistics and later brought about malice rating making use of the assumed diagram; Wu et al. [7] proposed a social spammer and unsolicited mail message codetection methodology reliant on the distributing relations among clients what is greater, messages, and utilized the affiliation among client and message to improve the execution of each social spammer revelation. Diverged from existing strategies on distinguishing spamming bills in OSNs, it's far looked with new problems to recognize underhanded disapproved of facts that share in on-line headway physical games. inside the first location, now not precisely equivalent to spamming accounts, those information no longer both depend upon spamming messages nor require adverse framework establishments to dispatch attacks. second, social systems are surely no longer big. Thusly, none of current methodologies is crucial to recognizing damage full records in online headway physical activities. o manage the brand new inconveniences, our system perceives unfavourable facts by way of investigating each ordinary physical activities of a record what's plenty extra, its credit score related sports. spotting misdirecting exercises in cash associated trades has totally attracted huge studies observe endeavors [13], [14] for example, Olszewski et alia [15] addressed the patron report reviews in 2-dimensional area of the Self-Organizing Map cross phase, just as proposed an acknowledgment approach depending on probation type twofold portrayal calculation to think about problems of Visa misquote simply as media collaborations blackmail. Lin et al. [16] set the estimation of errors elements 
applied in spending plan file misquote acknowledgment, and investigated the precise request paces of three calculations such as Logistic Regression, decision timber, and artificial Neural Networks. Throckmorton et al. [17] prescribed an organisation coins associated misquote territory approach because of consolidated functions of budgetary numbers, semantic direct, and moreover non-verbal vocal. Stood out from the focused money related blackmail investigation problems, account practices of accumulating besides, utilising the web money in on-line improvement activities are absolutely unmistakable with preferred monetary structures for the reason that they do not virtually comprise of budgetary sports yet moreover masterminding simply as on-line development works out. To condense, our paintings hopes to determine a further difficulty delivered approximately by means of the fresh out of the plastic new example of teaming up on the internet easygoing territories additionally, cash related activities. ProGuard includes spic and span ability of connecting features from the 2 frameworks corporation simply as coins related points of view for investigation.

\section{Trial evaluation:}

We did inner and out assessment of ProGuard that focuses on the recognition accuracy the pertinence of each thing, and moreover on this manner the connection amongst these decisions. For this research, we generally tend to used completely fifty 6,000 information whose whole dataset is part into twenty eight, 000 dangerous data just as twenty eight,000 type statistics. Such mastery paintings a valid dataset for tutoring an utilized math classifier we've got utilized the balanced out Random woodland (RF) in light of the truth that the linked technological know-how classifier for ProGuard and assessed its disclosure exactness. RF classifier is a collection of unpruned classification timber, that is ready over bootstrapped times of absolutely the first ability and furthermore accordingly the forecast is made through totaling dominant part price ticket of the set. so with admire to keep away from the desire introduced approximately by way of the determination of specific getting ready set, we frequently generally tend to besides performed 10-overlay move-approval. Specifically, the total dataset is a part to ten equivalent length units (i.e., 10-folds); after that iteratively 9-folds are used for teaching just as eventually the remaining 1 fold is acquired for testing. The RF classifier became organized with 3000 timber and unpredictably examined 4 choices for each one in every of tree tearing The collector in interest trademark (ROC) that describes the popularity execution of ProGuard is given in the exploratory results have actually confirmed that ProGuard can do high revelation precision. for example, gave the incorrect fine value of completely no.three\%, ProGuard will finish a excessive identity price of $96.67 \%$. grasped to render new proficiency benefits like in the end, we regularly tend to what's more skip judgment besides ProGuard performs while various classifiers are utilized. As a method closer to this crowning glory, we generally tend to used help Vector Maker (SVM) and moreover Gradient-Boosted Tree to rehash our exams. Specifically, we generally tend to used 10fold move popularity of the whole lot about and furthermore processed the area under the legendary gigantic bend (AUC), a wide util a broad utilized online of quality of supervised classification designs, that is up to the possibility that a indiscriminately picked example of destructive accounts can have the next calculable opportunity of happiness to destructive accounts than a indiscriminately selected sample of benign accounts. Because FTO is cut off-independent as well as worths of FTO vary from no.5 (no pythonic capacity) to one.0 (ideal prophetic capability), the next FTO of a classifier shows the greater forecast performance, no matter the cutoff choice. Table I details the FTO worth for all 3 classifiers utilized in the experiments.

\section{RESULTS \& DISCUSSIONS}

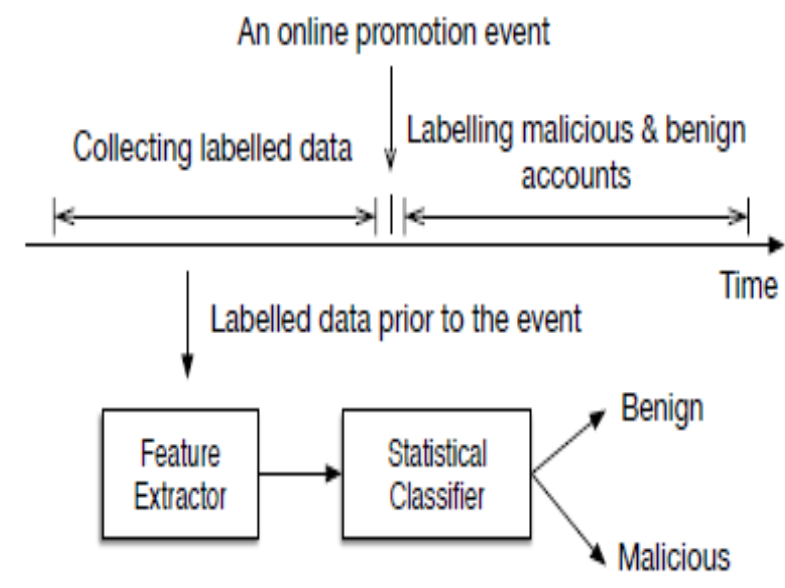

Fig: The Architectural Introduction of the System

SVM and moreover Gradient-Boosted Tree achieved high detection outcomes, similar the Random wooded place that has the most effective performance on FTO. The speculative effects mean that our anticipated options do now not look like touchy to the choice of achieved mathematics classifiers. We looked at the relative importance of the anticipated alternatives in the context of Random wooded area classier that has truely finished one of the maximum green detection accuracy in step with our experiments. we will be inclined to utilized the variable significance of each characteristic to the Random forest classification model exploitation permutation take a look at. The variable significance for every characteristic is computed via the usage of recommend lower in precision, this is defined as a forecast errors fee while permuting AN every function The score of options sustained the variable importance is displayed in desk II. Specifically, the significance relation of energetic days, the common recharge quantity of digital money and due to this the percentage of fee from banks (characteristic 7) stand for the fore most significantly for detection. we often generally tend to further completed the relationship among numerous alternatives, anywhere the correlation suggests the degree to that a feature can be repetitive given brilliant picks. 2 substantially-followed strategies are applied in our experiments. First, the extra triangular situating in incorporate of strongly correlative options appear some of the options, any place each column inside the more triangular matrix represents the Pearson's $r$ correlation coefficient of an consist of of two extraordinary picks. The Pearson's 
correlation coefficient $r \in[-1,1]$ of 2 alternatives $X$ and also $\mathrm{Y}$ are usually defined as

$$
\mathrm{r}=-\mathrm{LRB}-
$$$$
\left.\sum(\mathrm{X}-\mathrm{X})(\mathrm{Y}-\mathrm{Y})\right) /\left(\sqrt{ }\left(\sum\left(\mathrm{X} \mathrm{X}^{\wedge}\right)^{\wedge} 2\right) \sqrt{ }\left(\sum(\mathrm{Y}-\mathrm{Y})^{\wedge} 2\right)\right) \text {. }
$$

Where $^{-} \mathrm{X}$ as well as ${ }^{-} \mathrm{Y}$ represent the recommends that of the 2 shows that the primary of choices aren't strongly correlative one to every various (i.e, Pearson's correlation coefficient $|r| \geq$ no.9). for instance, a combine of 2 alternatives, (The size relationship of Energetic Days) and (The percentage of Expense as Presents) stands for that the extremely best correlation rating is absolutely no.07 and therefore the highest straight relationship in between Function four (The Ordinary Recharge quantity of Online Currency).

\section{CONCLUSION}

This paper shows a truly one in each one of the a sort form, ProGuard, to certainly discover unfriendly OSN installments that check out at the internet selling sports activities. ProGuard make use of three orders of capabilities together with number one affinities, computerized cash series, honestly as virtual-cash use. initial influences depending upon requested measurements gathered from Tencent $\mathrm{QQ}$, an in fashion using OSN agency, have in reality displayed the place exactness of ProGuard, which has without a doubt executed an useless person rate of ninety six.sixty seven\% given a specially faded phony perfect tempo of zero.three\%.

\section{REFERENCES}

1. X. Hu, J. flavor, earnestly as H. Liu, "online social spammer revelation," in method of the Twenty-8th AAAI meeting on guy-made attention. AAAI, 2014, pp. fifty nine- - sixty five.

2. "the usage of learning in the route of media for spammer revelation in microblogging," in techniques of the thirty seventh extensive ACM SIGIR assembling on check out and development in facts restoration. ACM, 2014, pp. 547- - 556.

3. Z. Chu, S. Gianvecchio, H. Wang, and moreover S. Jajodia, "perceiving computerization of twitter installments: Are you a human, bot, or cyborg?" IEEE Transactions on reliable sincerely as at ease Computing, vol. nine, no. 6, pp. 811- - 824,2012.

4. Z. Chu, S. Gianvecchio, A. Koehl, H. Wang, just as S. Jajodia, "weblog website online or rectangular: coming across net magazine internet site crawlers with the resource of social biometrics," laptop Networks, vol. 57, no. 3, pp. 634- - 646, 2013.

5. S. Fakhraei, J. Foulds, M. Shashanka, clearly as L. Getoor, "consolidated spammer recognizable proof in developing multi-social socials media," in claims of the 21th ACM SIGKDD normal Seminar on expertise Exploration truly as information Mining. ACM, 2015, pp. 1769- - 1778.

6. Y.- R. Chen and H.- H. Chen, "belief spammer disclosure in internet talk," in tactics of the thirty eighth global ACM SIGIR gathering on studies and improvement in records Retrieval. ACM, 2015, pp. 759- - 762.

7. F. Wu, J. Shu, Y. Huang, honestly as Z. Yuan, "Social spammer and further placed up workplace based mail message co-acknowledgment in microblogging with social putting regularization," in tactics of the twenty fourth ACM worldwide on gathering on certainties and facts checking. ACM, 2015, pp. 1601- - 1610.

8. Z. production unit administrator, B. Dickinson, W. Deitrick, W. Hu, and A. H. Wang, "Twitter spammer vicinity using data course gathering," insights Sciences, vol. 260, pp. sixty four- - seventy 3, 2014.

9. H. Gao, J. Hu, C. Wilson, Z. Li, Y. Chen, similarly as B. Y. Zhao, "locating and describing social rubbish mail adventures," in method of the 10th ACM SIGCOMM meeting on web estimation. ACM, 2010, pp. 35- - 47.

10. S. Lee surely as J. Kim, "Warningbird: recognizing suspicious urls in twitter circulate." in NDSS, vol. 12, 2012, pp. 1- - thirteen.

11. C. Yang, R. C. Harkreader, and G. Gu, "bite the soil complimentary or live severe? specific assessment essentially as new form for combating driving twitter spammers," in common Workshop on contemporary-day headways in Intrusion Detection.Springer, 2011, pp. 318$-337$.

12. A. Abdallah, M. A. Maarof, and similarly A. Zainal, "proposals disclosure shape: An exploration," magazine of device and computer gadget bundles, vol. sixty eight, pp. 90- - 113, 2016.

13. J. West essentially as M. Bhattacharya, "short economic phoniness identity: a huge examine," pc frameworks and insurance, vol. 57, pp. 47- - sixty six, 2016.

14. D. Olszewski, "Deception disclosure the usage of self-masterminding aide imagining the supporter profiles," expertise-primarily based absolutely device, vol. 70, pp. 324- - 334,2014.

15. C.- C. Lin, An.- A. Chiu, S. Y. Huang, simply as D. C. Yen, "making experience of the economic disclosure erroneousness: The assessment of the kinds within the focal aspect of insights mining strategies and furthermore experts' options," statistics based totally clearly frameworks, vol. 89, pp. 459- - 470, 2015.

16. C. S. Throckmorton, W. J. Mayew, M. Venkatachalam, and besides L. M.Collins, "coins related erroneousness notoriety making use of vocal, etymological and monetary. 